\title{
Biomechanically Constrained Groupwise US to CT Registration of the Lumbar Spine*
}

\author{
Sean Gill ${ }^{1}$, Parvin Mousavi ${ }^{1}$, Gabor Fichtinger ${ }^{1}$, Elvis Chen ${ }^{1}$, \\ Jonathan Boisvert ${ }^{1,2}$, David Pichora ${ }^{1}$, and Purang Abolmaesumi ${ }^{1}$ \\ 1 Queen's University, Kingston, ON, Canada \\ ${ }^{2}$ Institute for Information Technology, National Research Council Canada, Ottawa, \\ ON, Canada \\ pmousavi@cs.queensu.ca
}

\begin{abstract}
Registration of intraoperative ultrasound (US) with preoperative computed tomography (CT) data for interventional guidance is a subject of immense interest, particularly for percutaneous spinal injections. We propose a biomechanically constrained group-wise registration of US to CT images of the lumbar spine. Each vertebra in CT is treated as a sub-volume and transformed individually. The sub-volumes are then reconstructed into a single volume. The algorithm simulates an US image from the CT data at each iteration of the registration. This simulated US image is used to calculate an intensity based similarity metric with the real US image. A biomechanical model is used to constrain the displacement of the vertebrae relative to one another. Covariance Matrix Adaption - Evolution Strategy (CMA-ES) is utilized as the optimization strategy. Validation is performed on CT and US images from a phantom designed to preserve realistic curvatures of the spine. The technique is able to register initial misalignments of up to $20 \mathrm{~mm}$ with a success rate of $82 \%$, and those of up to $10 \mathrm{~mm}$ with a success rate of $98.6 \%$.
\end{abstract}

\section{Introduction}

Spinal injections for back-pain management are carried out on a frequent basis in hospitals and radiological clinics. Currently, these procedures are performed under fluoroscopy or CT guidance in specialized interventional radiology facilities, and thus incur a major financial burden on the healthcare system. Another drawback with the current practice is patient and surgeon exposure to X-ray radiation. The goal of this research is to design a spine intervention system that uses US for guidance. This would greatly reduce the exposure of both the patient and the physician to ionizing radiation and allow the procedure to be performed outside of a specialized facility. The use of only US for guidance has its own difficulties. In particular, due to the significant level of occlusion in spinal US images, it can be difficult to accurately identify the appropriate injection site. For this reason, in this paper, we consider the fusion of intraoperative US with preoperative CT as a means of guidance for spinal injections (Figure 1).

\footnotetext{
* This work has been partially funded by NSERC and CIHR. Special thanks to A. Lang, K. Wang, S. Lyman, J. Lazazzera and M. Kunz.
} 


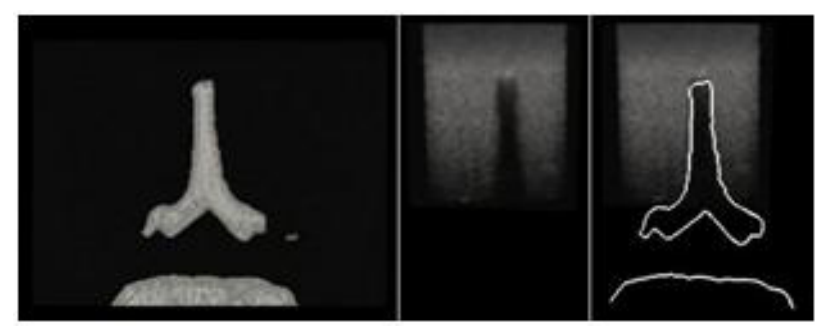

Fig. 1. Axial slice from a CT volume of the spine phantom (left), corresponding US slice (center) and an overlay of the CT bone contours with the corresponding US slice (right)

Point- and surface-based registration of US to CT data often require manual intervention and segmentation of US data, which is time consuming and susceptible to errors. To avoid these problems, we chose to focus on automated intensitybased registration methods. Among previously proposed intensity based methods for registration of US to CT data, Winter et al. 1 propose to define the bone surface in CT that is visible in an US image, with the similarity calculated as the US pixel intensities overlapping the surface. This requires a priori knowledge of the direction and orientation of the US probe. Penney et al. 2] present a method where voxel values in the US and CT data are converted to the probability of representing a bone edge. In order to have clinically relevant probabilities, a large set of prior CT and US images would have to be manually segmented.

In Wein et al. 34, density information from CT data is used to iteratively simulate an US image throughout the registration process, thereby optimizing the simulation as the registration proceeds. This has the benefit of not requiring any previous knowledge of the orientation of the US probe. Although the simulation is a simplified take on the physics of ultrasound beam propagation, it is sufficient for registration purposes. This algorithm is extended in Shams et al. [5] to create a more realistic simulation for training physicians and technicians in the use of US imaging. The technique requires preprocessing to create a scatter volume of the CT data using the Field II simulator, which remains time consuming. In Reichl et al. [6] and Kutter et al. 7] the US simulation and registration is implemented in GPU, resulting in a dramatically decreased algorithmic run time. Gill et al. 8, propose an extension of the work from Wein et al. [34, a groupwise US to CT registration of vertebrae L3 to L5. The algorithm allows free motion of the vertebrae and registers all three simultaneously. The drawback of this approach is that free motion of vertebrae during registration can lead to biologically unrealistic alignments.

Here we propose an algorithm that extends the groupwise registration presented in Gill et al. 8]. The algorithm allows independent motion of the vertebrae, but constrains their motion based on a well known biomechanical model. The registration is tested on a phantom printed using a surface model of a patient's spine which preserves a clinically realistic curvature of the spine. We present the results of the registration with various weights for the fusion of the biomechanical model with the intensity-based similarity metric. 


\section{US to CT Registration}

The registration workflow can be seen in Figure 2, The CT volume is initially cut into sub-volumes, each containing a single vertebra. Voxels in the sub-volumes corresponding to bone from an external vertebra are masked. The registration treats each vertebra independently, allowing for six degrees of freedom. All vertebrae are registered simultaneously, resulting in an optimization with $n \times 6$ parameters, where $n$ is the number of vertebrae being registered. After the transformations are applied, the sub-volumes are reconstructed into a single volume. For any overlapping voxels the maximum intensity is selected for the reconstructed volume, thus preserving bone structure. Any gaps not in the final volume are filled with a default value approximating the intensity of soft tissue in CT. The US simulation is applied to this reconstructed volume. CMAES is used as the optimization strategy, as Gill et al. [8] found it to be robust for US to CT simulation and registration.

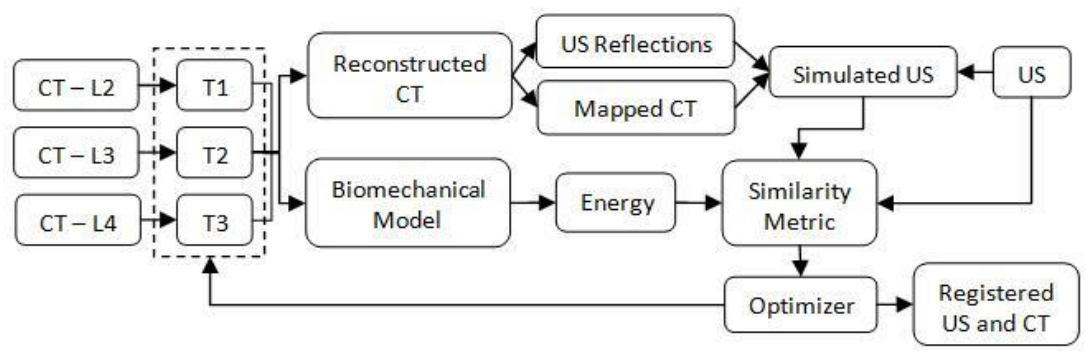

Fig. 2. Workflow of the biomechanically constrained groupwise US to CT registration

There are three distinct steps in the simulation of US from CT: The Simulation of the US reflection from CT, the mapping of the $\mathrm{CT}$ values to those found in US, and calculations of the weights for these to images and a bias. The weights are chosen so that the simulation best represents the real US image.

The simulated ultrasound reflections model the ultrasound beam passing through the tissue as a ray. The assumption is made that the CT intensities (in Hounsfield units) can be related to the acoustic impedance values used to calculate ultrasound transmission and reflection. The simulated beam passes through each column of the volume. The transmission and reflection of the beam is calculated at each voxel based of the following equations,

$$
\begin{gathered}
\Delta r(x, y, d)=\left(d^{T} \nabla \mu(x, y)\right) \frac{|\nabla \mu(x, y)|}{(2 \mu(x, y))}, \\
\Delta t(x, y)=\left(1-\left(\frac{|\nabla \mu(x, y)|}{(2 \mu(x, y))}\right)^{2},\right. \\
r(x, y)=I(x, y-1) \Delta r(x, y, d),
\end{gathered}
$$




$$
I(x, y)=\left\{\begin{array}{ll}
I(x, y-1) \Delta t(x, y), & |\nabla \mu(x, y)|<\tau \\
0, & |\nabla \mu(x, y)| \geq \tau
\end{array},\right.
$$

where $d$ is the direction of the US beam, $\mu$ is the intensity of the CT image, $\Delta R$ is reflection coefficient, $r$ is the simulated reflection intensity, $\Delta t$ is the transmission coefficient, $\tau$ is the threshold for full reflection and $I$ is the intensity of our simulated US beam. Any gradient value greater than a set threshold (450 h.u. in our simulations) causes full reflection of the US beam intensity at that point, setting the incoming US beam intensity for all subsequent points on the scan line to zero. A log-compression is applied to the simulated reflection image to amplify small reflections,

$$
r(x, y)=\frac{\log (1+a r(x, y))}{\log (1+a)} .
$$

The CT intensities are mapped to values closer to those corresponding to the tissues in the US data using an approximation of the curve presented in Wein et al. 34,

$$
p(x, y)=1.36 \mu(x, y)-1429 \text {. }
$$

The final step of the US simulation is the weighting of the simulated US reflection, the mapped CT and a bias term. A least-squares optimization is used to calculate the weights, such that the values in the simulation best match the corresponding intensities in the real US volume. The final simulation is calculated as,

$$
f(x, y)= \begin{cases}\alpha p(x, y)+\beta r(x, y)+\gamma, & I(x, y)>0 \\ 0, & I(x, y)=0\end{cases}
$$

where $f$ is the simulated US image and $\alpha, \beta, \gamma$ are the weights for their respective images. We do not include any voxels that are occluded in the simulation as part of the weight calculation. All occluded voxels in the US simulation are set to zero. Occluded voxels are identified as any voxel where the intensity of the incoming simulated US beam is zero. Similarity between the actual US image and simulated US image is calculated using the Linear Correlation of Linear Combination $\left(L C^{2}\right)$ metric presented by Wein et al. [3],

$$
L C^{2}=\frac{\sum(U(x, y)-f(x, y))^{2}}{N \times \operatorname{Var}(U)},
$$

where $N$ is the number of overlapping voxels between the US and CT images, and $U$ is the actual ultrasound image intensity. All voxels, including occluded voxels, are used in the calculation of the similarity metric.

\section{Biomechanical Model}

Allowing free motion of vertebrae during registration is not ideal as it does not distinguish between anatomically realistic orientations of vertebrae and orientations where the vertebrae are colliding, unreasonably oriented or far apart. We 
propose the use of a biomechanical model of the spine to constrain the registration and to favour anatomically acceptable alignments. The biomechanical model we use models the relation between the displacement of the intervertebral structures and the reaction forces and moments [9] :

$$
K=\left[\begin{array}{cccccc}
100 & 0 & 50 & 0 & -1640 & 0 \\
0 & 110 & 0 & 150 & 0 & 580 \\
50 & 0 & 780 & 0 & -760 & 0 \\
0 & 150 & 0 & 1.48 E 5 & 0 & -8040 \\
-1640 & 0 & -760 & 0 & 1.52 E 5 & 0 \\
0 & 580 & 0 & -8040 & 0 & 1.53 E 5
\end{array}\right]\left[\mathrm{Nmm} \mathrm{rad}^{-} 1\right]
$$

where $K$ is the stiffness matrix representing the intervertebral structures. This stiffness matrix is multiplied with a vector $x$ representing the change in translation and rotation of the intervertebral link. For our case, $x$ is calculated as the relative transform between two consecutive vertebrae. Each vertebra is expected to have no change in rotational orientation and no translation along the $\mathrm{x}$ and y axes. The expected translation along the coronal axis is defined as the mean distance between the centers of consecutive vertebrae in the patient's CT data. Note that this is meant as an approximation of the vertebral resting position.

The energy of the system based on the relative transformations between the vertebrae is calculated using the general spring equation,

$$
U=\frac{1}{2}\left(x^{T} K x\right) .
$$

The energy is calculated across all vertebrae and normalized based on the energy of a maximum misalignment $(15 \mathrm{~mm}$ translation along each axis and 15 rotation about each axis),

$$
E=\frac{\left(U_{\mathrm{L} 2, \mathrm{~L} 3}+U_{\mathrm{L} 3, \mathrm{~L} 4}\right)}{2 \times U_{\max }},
$$

where $E$ is the normalized energy of the system, $U_{\mathrm{L} 2, \mathrm{~L} 3}$ and $U_{\mathrm{L} 3, \mathrm{~L} 4}$ represent the energy of the model calculated from the relative transforms between L2-L3 and L3-L4, respectively, and $U_{\max }$ is the energy of the maximum misalignment.

This normalized energy is then combined with the $L C^{2}$ metric to give the Biomechanically Constrained Linear Correlation of Linear Combination $\left(B C L C^{2}\right)$,

$$
B C L C^{2}=L C^{2}-\sigma E,
$$

where $\sigma$ is a user defined weight used to blend the biomechanical model measure with the $L C^{2}$ intensity based measure.

\section{Results}

Registration accuracy was validated on a patient-mimicking phantom of the lumbar spine. Vertebrae L1 to L5 were segmented from patient CT data using ITK-SNAP. The segmented data was converted to a surface model and the spine was printed using a Cimetrix 3D shape printer (Cimetrix Solutions, Oshawa, ON, 
Canada). In this model, the natural curvature of the spine is preserved between the patient CT and the printed phantom. The phantom was filled with an agargelatine recipe [10, designed to simulate the appearance of soft tissue in US. A high-resolution CT volume $(0.46 \mathrm{~mm} \times 0.46 \mathrm{~mm} \times 0.625 \mathrm{~mm})$ and an US volume were acquired. The US volume was reconstructed from a freehand sweep using an L14-5/38 linear-array transducer (Ultrasonix, Richmond, BC, Canada) operating at $6.6 \mathrm{MHz}$ with a depth of $5.5 \mathrm{~cm}$. The probe was tracked using an Optotrack Certus System (Northern Digital Inc., Waterloo, ON, Canada) and calibrated using an N-wire US phantom 11. Registration was performed on a Dell Precision 690, $2 \times 2.33 \mathrm{GHz}$ Intel Xeon Quad-core CPU and 16GB of RAM.

The phantom CT and US data were aligned to the gold standard, determined by fiducial markers placed on the exterior of the phantom box. The registration was performed on vertebrae L2-L4 and the US volume was cropped to correspond, as seen in Figure 3. The middle vertebrae were chosen for registration as they contained overlap from other vertebrae at the facet joints.

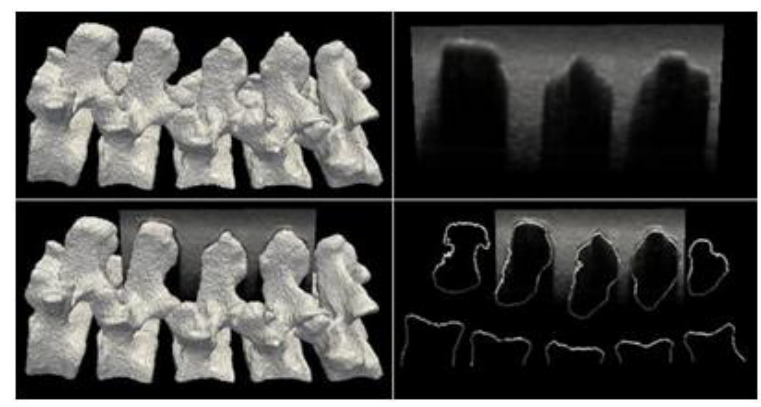

Fig. 3. Surface model of L1 to L5 (top left); US slice of L2 to L4 (top right). Overlaid US with surface model (bottom left) and corresponding extracted slice (bottom right).

One hundred registrations of the CT and US data were performed on the phantom with initial misalignment ranging from $0-20 \mathrm{~mm}$ Target Registration Error (TRE). The CT volume was misaligned by a random transform chosen from a uniform distribution of $10 \mathrm{~mm}$ translation along each axis and 10 rotation about each axis. Each vertebra was further misaligned by individually applying a random transform using a uniform distribution of $5 \mathrm{~mm}$ translation along each axis and 5 rotation about each axis. We chose this distribution to ensure that the registration capture range is greater than that of a clinical setting. The registrations were repeated for $\sigma$ values of $0,0.5,1$ and 2 .

Registration accuracy was determined by its ability to recover to the gold standard alignment, and is reported as the mean TRE calculated from the misalignment of the eight corners of the volume bounding box. Registration results are presented in Table 1 and an example of the initial misalignment and the final registration is displayed in Figure 4. TRE is calculated for each vertebra and the overall error is calculated as the mean error across the entire volume. A registration is considered failed if the final TRE is greater than $3 \mathrm{~mm}$. 
Table 1. Final TRE for vertebrae L2-L4 and the mean error of the volume are presented for all successful registrations. Success rate (SR) is defined as the percentage of registrations where the overall final TRE is less than $3 \mathrm{~mm}$. SR is presented for all registration and for registrations with initial TRE of less than $10 \mathrm{~mm}$.

\begin{tabular}{ccccccc}
\hline$\sigma$ & $\begin{array}{c}\text { L2 } \\
(\mathrm{mm} / \mathrm{std})\end{array}$ & $\begin{array}{c}\text { L3 } \\
(\mathrm{mm} / \mathrm{std})\end{array}$ & $\begin{array}{c}\text { L4 } \\
(\mathrm{mm} / \mathrm{std})\end{array}$ & $\begin{array}{c}\text { Overall } \\
(\mathrm{mm} / \mathrm{std})\end{array}$ & SR (\%) & $\begin{array}{c}\text { SR: } \\
\text { ITRE }<10 \mathrm{~mm}(\%)\end{array}$ \\
\hline 0.0 & $1.49 / 0.57$ & $1.83 / 1.02$ & $1.93 / 0.85$ & $1.75 / 0.46$ & 61.0 & 77.8 \\
0.5 & $1.82 / 0.36$ & $1.62 / 0.54$ & $2.47 / 0.60$ & $1.97 / 0.30$ & 82.0 & 98.6 \\
1.0 & $2.19 / 0.34$ & $1.79 / 0.46$ & $3.04 / 0.44$ & $2.34 / 0.21$ & 80.0 & 94.4 \\
2.0 & $2.46 / 0.21$ & $1.58 / 0.28$ & $4.12 / 0.38$ & $2.71 / 0.13$ & 72.0 & 84.8 \\
\hline
\end{tabular}

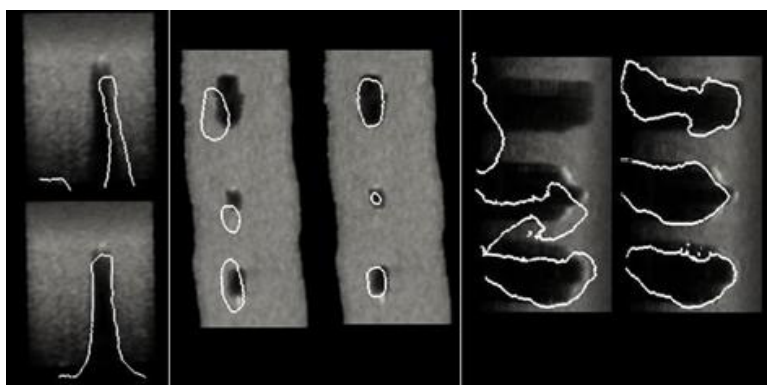

Fig. 4. Transverse (Left), Coronal (Center) and Sagittal (Right) slices of the original US volume overlaid with the bone contours in the misaligned CT volumes and in the registered CT volumes. The transverse slice is taken from the center vertebra L2 (Top).

\section{Discussion and Conclusion}

In this work we presented an US to CT registration technique for the lumbar spine that successfully registered $98.6 \%$ of volumes with initial misalignments of up to $10 \mathrm{~mm}$. The registration algorithm applied iterative US simulation from CT images while allowing independent motion of each vertebra. A biomechanical model was introduced to represent the intervertebral link and the system energy was calculated based on the relative transforms between the vertebrae. Integration of a biomechanical model to constrain the registration greatly improved the consistency of the algorithm. When the biomechanical model was combined with the $L C^{2}$ metric with a $\sigma$ weight of 0.5 , the algorithm produced the best results: an overall accuracy of $1.97 \mathrm{~mm}$, a failure rate across all registrations of $18 \%$ and a failure rate, for registration with initial misalignment less than $10 \mathrm{~mm}$, of $1.4 \%$. The registration technique was tested on patient mimicking phantom that were faithful in representing a realistic curvature of the spine (L1-L5). One hundred tests were performed where each vertebrae was misaligned between $0 \mathrm{~mm}$ and $20 \mathrm{~mm}$. The tests were repeated for various weights for integrating the biomechanical model. Using a $\sigma$ value of $0.5,82 \%$ of all registrations were successful, while $98.6 \%$ of tests with initial misalignment of less than $10 \mathrm{~mm}$ were successfully registered. Increasing the weight of the biomechanical model, to $\sigma$ equal to 
1 or higher, increased the failure rate of the registration. Similarly we observe that while the inclusion of a biomechanical model improves the success rate of a registration, it can also decrease the accuracy of those successful registrations. This can be explained by the fact that the model used is an approximation and not specific to the given patient. The TRE values presented are calculated based on the misalignment of the bounding box corners for each sub-volume. We believe this is a worst case estimate of the error and the error found at the facet joints (approximately centre of the box) will be lower.

The mean run time of our registrations was $57.4 \mathrm{~min}$. To reduce this to a more clinically acceptable run time, we have begun the implementation of this algorithm in GPU. Our preliminary implementation, running in CUDA 1.1 on an Nvidia GTX285 graphics processor, reduced the registration runtime to $537 \mathrm{~s}$, a $6.4 \mathrm{x}$ improvement. In our future work we plan to optimize the GPU implementation and extend the registration to the full lumbar spine, L1-L5. In addition, we also plan to test the registration on real patient data.

\section{References}

1. Winter, S., Brendel, B., Pechlivanis, I., Schmieder, K., Igel, C.: Registration of ct and intraoperative 3-d ultrasound images of the spine using evolutionary and gradient-based methods. Evol. Comput. 12(3), 284-296 (2008)

2. Penney, G., Barratt, D., Chan, C., Slomczykowski, M., Carter, T., Edwards, P., Hawkes, D.: Cadaver validation of intensity-based ultrasound to ct registration. Medical Image Analysis 10(3), 385-395 (2006)

3. Wein, W., Khamene, A., Clevert, D.-A., Kutter, O., Navab, N.: Simulation and fully automatic multimodal registration of medical ultrasound. In: Ayache, N., Ourselin, S., Maeder, A. (eds.) MICCAI 2007, Part I. LNCS, vol. 4791, pp. 136143. Springer, Heidelberg (2007)

4. Wein, W., Brunke, S., Khamene, A., Callstrom, M.R., Navab, N.: Automatic ctultrasound registration for diagnostic imaging and image-guided intervention. Medical Image Analysis 12(5), 577-585 (2008)

5. Shams, R., Hartley, R.I., Navab, N.: Real-time simulation of medical ultrasound from CT images. In: Metaxas, D., Axel, L., Fichtinger, G., Székely, G. (eds.) MICCAI 2008, Part II. LNCS, vol. 5242, pp. 734-741. Springer, Heidelberg (2008)

6. Reichl, T., Passenger, J., Acosta, O., Salvado, O.: Ultrasound goes gpu: real-time simulation using cuda, vol. 7261, p. 726116. SPIE (2009)

7. Kutter, O., Karamalis, A., Wein, W., Navab, N.: A gpu-based framework for simulation of medical ultrasound, vol. 7261, p. 726117. SPIE (2009)

8. Gill, S., Mousavi, P., Fichtinger, G., Pichora, D., Abolmaesumi, P.: Group-wise registration of ultrasound to ct images of human vertebrae, vol. 7261, p. 726110 . SPIE (2009)

9. Desroches, G., Aubin, C.E., Sucato, D.J., Rivard, C.H.: Simulation of an anterior spine instrumentation in adolescent idiopathic scoliosis using a flexible multi-body model. Med. Bio. Eng. Comput. 45(8), 759-768 (2007)

10. Madsen, E.L., Hobson, M.A., Shi, H., Varghese, T., Frank, G.R.: Tissue-mimicking agar/gelatin materials for use in heterogeneous elastography phantoms. Phys. Med. Biol. 50(23), 5597-5618 (2005)

11. Chen, T.K., Thurston, A.D., Moghari, M.H., Ellis, R.E., Abolmaesumi, P.: A realtime ultrasound calibration system with automatic accuracy control and incorporation of ultrasound section thickness, vol. 6918, p. 69182A. SPIE (2008) 